\title{
Identifying quantum topological phases through statistical correlation
}

\author{
Hao Wang, ${ }^{1}$ B. Bauer, ${ }^{2}$ M. Troyer, ${ }^{2}$ and V. W. Scarola ${ }^{1}$ \\ ${ }^{1}$ Physics Department, Virginia Tech, Blacksburg, Virginia 24061, USA \\ ${ }^{2}$ Theoretische Physik, ETH Zurich, CH-8093 Zurich, Switzerland
}

(Received 2 October 2010; revised manuscript received 7 February 2011; published 14 March 2011)

\begin{abstract}
We theoretically examine the use of a statistical distance measure, the indistinguishability, as a generic tool for the identification of topological order. We apply this measure to the toric code and two fractional quantum Hall models. We find that topologically ordered states can be identified with the indistinguishability for both models. Calculations with the indistinguishability also underscore a key distinction between symmetries that underlie topological order in the toric code and quantum Hall models.
\end{abstract}

DOI: 10.1103/PhysRevB.83.115119

PACS number(s): 03.65.Vf, 73.43.-f, 05.30.Pr

\section{INTRODUCTION}

Conventional types of quantum order can be characterized by local symmetries. Topological quantum order, in contrast, defies characterization by local operators. ${ }^{1}$ Topological order owes its structure to nonlocal properties and therefore depends on the surface on which it is placed. Examples of topologically ordered quantum states include the ground state of Kitaev's toric code model ${ }^{2}$ and quantum Hall states. ${ }^{3}$ Such states are not characterized by simple, local order parameters. Analyses based on system entanglement entropy and other nonlocal properties have been used to study these states. ${ }^{4,5}$

Wave functions (e.g., the Laughlin state ${ }^{6}$ ) capture the essential properties of some of the fractional quantum Hall $(\mathrm{FQH})$ states. Indeed, full microscopic analyses are typically done with wave functions in efforts to accurately capture the low energy physics of insoluble quantum Hall models. ${ }^{7,8}$ These wave functions, in turn, describe incompressible quantum liquids with no simple local order parameter.

Kitaev has constructed exactly soluble spin models, the toric code $^{2}$ and honeycomb ${ }^{9}$ models, to analytically probe topological order. These two-dimensional models exhibit onedimensional string symmetries that underlie topological order. A comparison between symmetries in these spin models and certain symmetries of the $\mathrm{FQH}$ regime ${ }^{10}$ has been recently drawn. ${ }^{11}$ We ask if one can use numerical methods to generically identify and compare topological order in both types of models.

The indistinguishability ${ }^{12-14}$ was recently proposed ${ }^{15}$ as a tool to probe complex quantum states. The indistinguishability is a statistical distance measure that yields the probability of making an error in an $n$-particle measurement in an attempt to distinguish two states. Reference 15 used explicit calculations on one-dimensional spin models to test if this measure can act as an effective nonlocal order parameter to identify quantum states. Scaling relations were found in transitions between states in the quantum Ising model and the bilinearbiquadratic Heisenberg chain without making recourse to local order parameters. Phases and phase transitions were instead identified using ansatz states.

In this paper we study the indistinguishability as a method to identify topological quantum order in two-dimensional models. We study the toric code and models of the FQH regime. We find that in the toric code the indistinguishability reveals distinct topological sectors and the one-dimensional nature of the symmetries defining each sector. We then use the indistinguishability to underscore a key difference between topological order in the toric code and the $\mathrm{FQH}$ regime. By diagonalizing models of the $\mathrm{FQH}$ effect we show that distinct topological sectors (and distinct $\mathrm{FQH}$ states in general) differ in that symmetry operators must span the entire system rather than just one-dimensional operators. The measure can be used to identify mechanisms of topological ordering in more nontrivial models where symmetries and a complete characterization of states have not been performed.

In Sec. II we review the indistinguishability as a measure of distinct quantum orders. In Sec. III we examine the scaling behavior of the indistinguishability in the toric code. In Sec. IV we examine the scaling of the indistinguishability in $\mathrm{FQH}$ models of the Laughlin, charge density wave ${ }^{16}$ (CDW), and Moore-Read ${ }^{17}$ states. We summarize in Sec. V with a comparison of results for both sets of models.

\section{INDISTINGUISHABILITY}

The indistinguishability is based on a quantum information measure of quantum state distinguishability. ${ }^{12-14}$ We define the indistinguishability $I_{n}(\mathrm{~A}: \mathrm{B})$ of two $N$-particle states, $\Psi_{\mathrm{A}}$ and $\Psi_{\mathrm{B}}$, as the probability of making an error in distinguishing the two states with an $n$-particle measurement:

$$
I_{n}(\mathrm{~A}: \mathrm{B})=\frac{1}{2}-\frac{1}{4} \operatorname{Tr}\left|\rho_{\mathrm{B}}^{(n)}-\rho_{\mathrm{A}}^{(n)}\right|,
$$

where $\operatorname{Tr}|\Omega|$ is the trace norm of $\Omega$ and $\rho^{(n)}=\operatorname{Tr} r_{N-n}(\rho)$ is the $n$-particle reduced density matrix and $\mathrm{T} r_{N-n}$ denotes the partial trace over $n$ particles. Interpreting the density matrix as a probability distribution, the last term in Eq. (1) can be identified with a well-known statistical distance measure, the Kolmogorov distance. When $I_{n}$ is zero, two states are distinguishable and the ansatz state $\Psi_{\mathrm{A}}$ is a poor approximation to $\Psi_{\mathrm{B}}$. However, when it is nonzero, there is a finite probability that an $n$-particle measurement cannot distinguish the two states. $I_{n}=1 / 2$ corresponds to the maximum indistinguishability, implying two identical states with unitary wave function overlap when $n=N$. In contrast to the entanglement entropy used in the FQHE regime, here the state indistinguishability yields a single number that quantifies the ability of an optimally chosen set of $n$-particle correlators to distinguish two states. ${ }^{12,13} 1-I_{n}$ gives the probability that an optimally 
chosen correlation function involving at most $n$ particles will be able to distinguish the two states.

We use $I_{n}$ to quantify the degree of indistinguishability of two states imposed by underlying correlators in an $N$-particle system. In cases where a small constant value of $n \sim O(1)$ suffices to characterize the correlators (i.e., two states can be distinguished locally), we define $I_{n}$ to be intensive in $N$. Such two states belong to the same $n$-particle correlator class if $I_{n}$ remains finite in the thermodynamic limit (i.e., as $N \rightarrow \infty$ ). On the other hand, if two states cannot be distinguished locally and therefore $n$ needs to scale with $N$, we define $I_{n}$ to be extensive. In this situation, we use the scaling of $n$ with $N$ to identify correlator classes. ${ }^{15}$ The precise scaling behavior of $n$ with $N$ [e.g., $n \sim O(N)$ or $n \sim O(\sqrt{N})$ ] provides us with a key feature to reliably distinguish phases.

In the following, we explore the scaling of the indistinguishability between topologically ordered quantum states.

\section{INDISTINGUISHABILITY IN THE TORIC CODE}

\section{A. Review of the toric code}

The toric code Hamiltonian was constructed as an exactly soluble model with a topologically ordered ground state and anyonic excitations. ${ }^{2,18}$ We briefly review the model and discuss its symmetry properties. The model is given by

$$
H_{T}=-\sum_{v} \prod_{j \in v} \sigma_{j}^{x}-\sum_{p} \prod_{j \in p} \sigma_{j}^{z},
$$

where $\sigma_{j}$ denotes Pauli matrices at sites $j$ on bonds of the square lattice. The first product is over the four sites surrounding the vertex $v$ while the second product is over the four sites around each plaquette $p$ (Fig. 1).

When placed on a torus, the model possesses two distinct one-dimensional $\mathbb{Z}_{2}$ symmetries. The operators $\prod_{j \in w^{\prime}} \sigma_{j}^{x}$ and $\prod_{j \in w} \sigma_{j}^{z}$ both commute with $H_{T}$ where $w^{\prime}$ is a loop along vertices and $w$ is a loop along bonds. These one-dimensional operators form closed loops around either cycle of a torus. They can be used to classify topological ground state sectors.

The ground state of the toric code is then given as the equal-amplitude superposition of vortex-free states:

$$
\left|\Psi_{i}\right\rangle=\sum_{|\xi\rangle \in \chi_{i}} f_{i}|\xi\rangle
$$

where $\chi_{i}$ are four spaces of such vortex-free configurations distinguished by the expectation value of the operator, $\prod_{i \in w} \sigma_{i}^{z}$, for two inequivalent noncontractible loops $w_{1}, w_{2}$ wrapping around the torus in two different directions. $f_{i}$ is a normalization factor which is equal for all sectors. By a vortex-free configuration, we mean a basis state $|\xi\rangle$ for which $\prod_{i \in \delta p} \sigma_{i}^{z}=+1$ for all plaquettes $\delta p$.

The toric code exhibits a phase transition under a magnetic field. This perturbation breaks the one-dimensional $\mathbb{Z}_{2}$ symmetries and can destroy topological order if it is strong enough. Numerical studies of ground state degeneracies and other indirect measures of topological order show a robust phase transition ${ }^{19}$ from the topologically ordered phase to a classically ordered phase with increasing magnetic field. A more recent study used a topological fidelity measure to
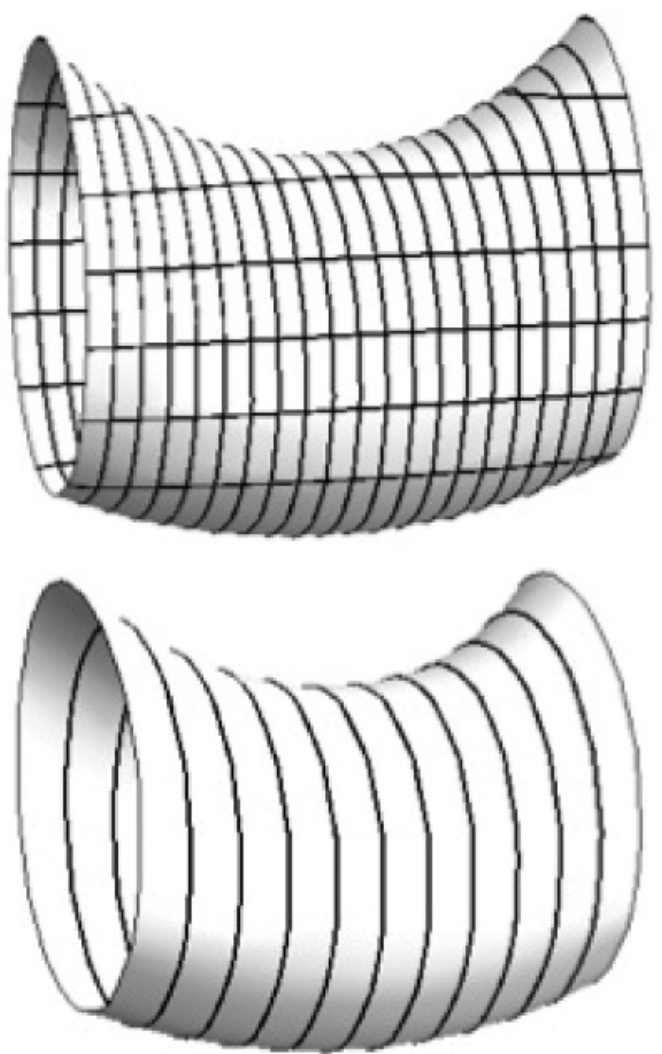

FIG. 1. Top: Section of torus depicting the two-dimensional basis of the toric code in real space. The sites sit on bonds between vertices to form a square lattice. Bottom: Section of torus depicting twodimensional basis of a single Landau level in real space. Basis states form a periodic array of rings in the Landau gauge.

observe the same transition by extracting finite size scaling information related to the one-dimensional $\mathbb{Z}_{2}$ symmetries. ${ }^{20}$

\section{B. Computed indistinguishability}

Given the above ground states of the toric code, we can analytically compute the indistinguishability between two topologically distinct sectors. We consider a square lattice $\mathcal{L}$ with spins located on $L$ bonds along each dimension and $N=2 L^{2}$ sites. A block $\mathcal{Q}$ of $n$ sites is chosen for calculating $I_{n}$. The remaining sites in the lattice are denoted as $\mathcal{R}$, i.e., $\mathcal{L}=\mathcal{Q} \cup \mathcal{R}$

To compute $I_{n}$ we must find $\varrho_{A}^{\mathcal{Q}}$ and $\varrho_{B}^{\mathcal{Q}}$, the reduced density matrices on a subset $\mathcal{Q} \subset \mathcal{L}$ for two different states $A$ and $B$, respectively. From Eq. (3) we find that the matrix elements of $\varrho_{A}^{\mathcal{Q}}-\varrho_{B}^{\mathcal{Q}}$ are given by

$$
\begin{aligned}
\left\langle v_{\mathcal{Q}}\left|\varrho_{A}^{\mathcal{Q}}-\varrho_{B}^{\mathcal{Q}}\right| w_{\mathcal{Q}}\right\rangle= & \sum_{\left|u_{\mathcal{R}}\right\rangle} f^{2}\left\{\sum_{\left|\xi_{1}\right\rangle,\left|\xi_{2}\right\rangle \in \chi_{A}}\left\langle v_{\mathcal{Q}} u_{\mathcal{R}} \mid \xi_{1}\right\rangle\left\langle\xi_{2} \mid w_{\mathcal{Q}} u_{\mathcal{R}}\right\rangle\right. \\
& \left.-\sum_{\left|\xi_{1}\right\rangle,\left|\xi_{2}\right\rangle \in \chi_{B}}\left\langle v_{\mathcal{Q}} u_{\mathcal{R}} \mid \xi_{1}\right\rangle\left\langle\xi_{2} \mid w_{\mathcal{Q}} u_{\mathcal{R}}\right\rangle\right\} \\
= & \sum_{\left|u_{\mathcal{R}}\right\rangle} f^{2}\left\{\delta_{A}\left(\left|v_{\mathcal{Q}} u_{\mathcal{R}}\right\rangle\right) \delta_{A}\left(\left|w_{\mathcal{Q}} u_{\mathcal{R}}\right\rangle\right)\right. \\
& \left.-\delta_{B}\left(\left|v_{\mathcal{Q}} u_{\mathcal{R}}\right\rangle\right) \delta_{B}\left(\left|w_{\mathcal{Q}} u_{\mathcal{R}}\right\rangle\right)\right\}
\end{aligned}
$$


Here, the states $\left|u_{\mathcal{R}}\right\rangle$ are all basis states on the sublattice $\mathcal{R}$, and $\delta_{A}(|\phi\rangle)=1$ if $|\phi\rangle \in \operatorname{span}\left(\chi_{A}\right), 0$ otherwise.

The above expression shows that if $\mathcal{Q}$ supports two inequivalent loops $w_{1}, w_{2}$, all sectors can be distinguished, as expected. If it only supports one such loop, only half of the sectors can be distinguished. If it does not wrap around the boundary, no sectors can be distinguished. $I_{n}$ is always either 0 or $1 / 2$. The above explicit calculation therefore shows that for a wisely chosen $\mathcal{Q}$, such that it wraps the boundary $\left(w_{1}, w_{2} \in \mathcal{Q}\right)$, correlators of size $n=O(\sqrt{N})$ are sufficient to reliably distinguish topological sectors.

We now ask how many measurements on randomly chosen spins are needed to distinguish topological sectors of the toric code. For simplicity, we consider only the case of distinguishing two sectors; i.e., we look for clusters wrapping around the torus in one nontrivial way. We seek the probability $\Pi(p)$ that a fraction $p$ of randomly chosen sites forms a cluster that wraps around the boundary. This is the problem of percolation with periodic boundary conditions. For this problem, it is well known that a critical $p_{c}$ exists such that in the thermodynamic limit, $\Pi=1$ for $p>p_{c}$ and $\Pi=0$ otherwise. The critical behavior is in fact identical to that of standard percolation with free boundary conditions. ${ }^{21,22}$ These well-known results from percolation theory indicate that in order to distinguish sectors of the ground state based on purely randomly chosen sites, a cluster size $n \sim O(N)$ is necessary.

A different situation occurs if we choose sites randomly, but as contiguous blocks. The probability for a contiguous cluster of size $n$ to wrap around the boundary, which we denote as $\varrho$, is given by

$$
\varrho(n)=\int_{p_{c}}^{1} d p \delta[N P(p)-n],
$$

where $P$ is the probability for one site to lie in the percolating cluster for a completely random choice of sites. We then have $I_{n}=[1-\varrho(n)] / 2$. We do not expect a sharp transition to appear in this quantity because there is a finite but exponentially small probability for a random block of size $n \geqslant \sqrt{N}$ to wrap around the boundary.

Scaling theory dictates that the behavior of $P$ in the thermodynamic limit and in the critical region is governed by $P \sim\left(p-p_{c}\right)^{\beta}$. The divergence of the correlation length is described by $\xi \sim\left(p-p_{c}\right)^{-v}$; however, on finite systems this is bounded by $L$ and therefore $\left(p-p_{c}\right) \sim L^{-1 / v}$. We then have $P \sim L^{-\beta / v}$ or, equivalently, a critical cluster size $n_{c} \sim L^{2-\beta v}=L^{D}$, where $D$ is the fractal dimension. If one were to grow only one cluster in the system, the probability for this cluster to percolate should increase rapidly at $n \sim O\left(L^{D}\right)$. In two dimensions, the value of $D$ is $91 / 48$. We can therefore expect that a contiguous cluster of size

$$
n \sim O\left(L^{91 / 48}\right)
$$

is sufficient to distinguish two sectors of the ground state.

To verify the above statement we compute $I_{n}$ explicitly using a direct-sampling Monte Carlo method. We draw the configurations of a cluster with $n$ connected sites from a uniform distribution and measure the probability for such

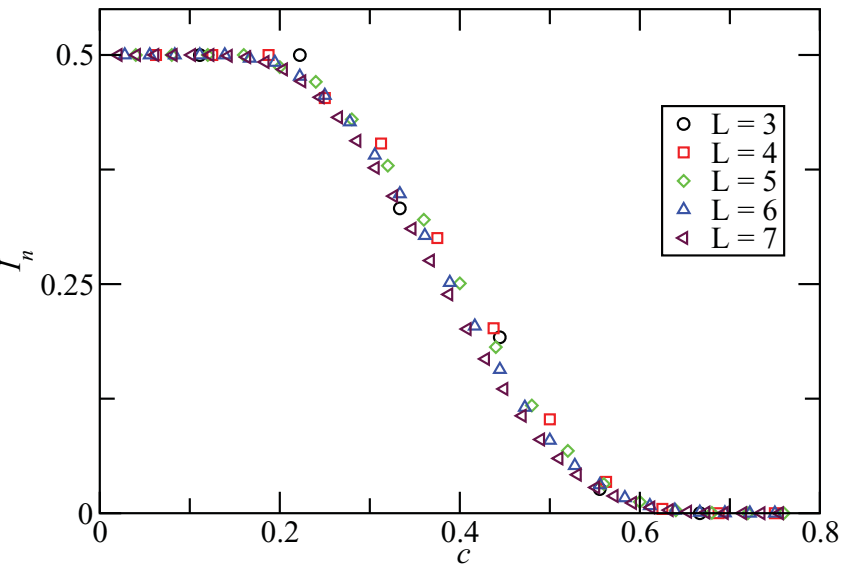

FIG. 2. (Color online) Plot of the $n$-particle indistinguishability versus $c=n / N$ for several different system sizes computed using Monte Carlo selection of random but contiguous collections of spins for the toric code on a two-dimensional periodic lattice with $N=2 L^{2}$ spins. The graph shows data collapse and a linear scaling of $n$ with system size $N$, in contrast to a $N^{1 / 2}$ scaling for properly chosen spins [Eq. (4)].

a cluster to support a loop wrapping around the boundary, $P($ loop $)=\varrho(n)$. The results of $I_{n}$ versus linear scaling ratio,

$$
c=\frac{n}{N},
$$

for several $L$ are shown in Fig. 2. The data collapse beyond a regime where finite-size effects are relevant, which agrees with the expected scaling $n \sim O\left(L^{D}\right)$. The difference between $L^{91 / 48}$ and $L^{2}$ is too small to be distinguished numerically.

We have thus shown that the indistinguishability reveals the size of the operators required to identify topological sectors. For suitably chosen blocks we find $n=O(\sqrt{N})$ whereas randomly chosen sites lead to $n=O(N)$. In the case where a random but contiguous choice of sites is made, the necessary block size is $n=O\left(L^{91 / 48}\right) . I_{n}$ thus yields topologically relevant information without requiring a precise identification of the nonlocal symmetries defining each sector. We now turn to models of the FQH effect that, in some limits, do not have exact solutions.

\section{INDISTINGUISHABILITY IN THE FRACTIONAL QUANTUM HALL REGIME}

We consider a two-dimensional electron gas (2DEG) on the surface of a torus under a magnetic field perpendicular to the surface. In a strong magnetic field, electrons occupy highly degenerate and energetically distinct Landau levels (LLs). At a fractional LL filling, $v$, ideal interaction models can generate topologically ordered ground states without defining local symmetries. Two examples include the Abelian Laughlin states $^{6}$ at $v=1 / 3$ from short range pair interactions ${ }^{23}$ and the non-Abelian Moore-Read state ${ }^{17}$ at $v=5 / 2$ from short range three-body interactions. ${ }^{24,25}$

In the torus geometry, these ground states are degenerate in multiple folds and show a finite energy gap from excited states, thereby suggesting topologically ordered states. A key question then arises. Is there a simple correlation function (e.g., 
a one-dimensional chain operator with $n \sim \sqrt{N}$ as for the toric code) that defines the topological sectors in the quantum Hall regime? A well-known result by Haldane ${ }^{10}$ discovered just such a symmetry. A product of translation operators around one toric cycle indeed connects distinct topological sectors. (For a review of this work and its connection to topological sectors see Ref. 26.) This center of mass operator requires all particles for its construction, in contrast to the chain operators identified in the toric code.

Peculiarities of the lowest LL basis require that a symmetry spanning at least one dimension must incorporate all particles. To see this we consider basis states on a section of the torus as shown at the bottom of Fig. 1. The Landau gauge basis states form periodic rings around the torus. Operators constructed from one-dimensional translations of these rings will encompass the entire system. One can show that there are no lowest LL basis states that are both orthogonal and localized in two dimensions. ${ }^{27}$ As a result, apparent one-dimensional symmetries must span all particles in two-dimensional lowest LL systems. $^{11}$

The apparent lack of true one-dimensional symmetries suggests that all $\mathrm{FQH}$ states are best characterized by $n \sim N$ correlation functions, i.e., wave functions, in systems without edges. We verify this assertion using the indistinguishability to compare a variety of different FQH states on the torus. We compare these states by first constructing generator models, diagonalizing these models, and then numerically computing $I_{n}$.

\section{A. Modeling fractional quantum Hall states}

We now review the Coulomb model of the FQH regime and ideal models that generate FQH states. Periodic boundary conditions for magnetic translational operators are imposed with a quantized flux $N_{\phi}$ through the unit cell. The magnetic length $\ell$ is taken as the unit length and the energy is in Coulomb units, $e^{2} / 4 \pi \epsilon \ell$. In the absence of LL mixing, the Hamiltonian of a 2DEG of $N$ particles interacting through the Coulomb interaction can be projected into the topmost LL with the filling factor $\tilde{v}=N / N_{\phi}:{ }^{25}$

$$
H_{c}=\frac{2}{N_{\phi}} \sum_{i<j} \sum_{\mathbf{q}} e^{-q^{2} / 2} e^{i \mathbf{q} \cdot\left(\mathbf{r}_{i}-\mathbf{r}_{j}\right)} \sum_{m=0}^{\infty} V_{m} L_{m}\left(q^{2}\right),
$$

where $V_{m}$ is Haldane's pseudopotential parameter ${ }^{23}$ and $L_{m}(x)$ is the Laguerre polynomial. The momenta $\mathbf{q}$ take discrete values suitable for the unit cell lattice. $\mathbf{r}_{i}$ is the guiding center coordinate of the $i$ th electron.

Our first example of the ideal state at $v=1 / 3$, the Laughlin state $\Psi_{L}$, is obtained as the densest zero-energy ground state of a short range interaction with only the pseudopotential $V_{1}$ nonzero in the above Coulomb Hamiltonian. Our second example of the ideal state at half-filled second LL $(v=5 / 2)$, the Moore-Read state $\Psi_{P f}$, is obtained as the densest zeroenergy ground state of a repulsive three-body potential: ${ }^{25}$

$$
H_{3}=-\sum_{i<j<k} S_{i, j, k}\left[\nabla_{i}^{4} \nabla_{j}^{2} \delta^{2}\left(\mathbf{r}_{i}-\mathbf{r}_{j}\right) \delta^{2}\left(\mathbf{r}_{j}-\mathbf{r}_{k}\right)\right],
$$

where $S_{i, j, k}$ is a symmetrizer. We can then compare these ideal states with the exact ground state of the Coulomb interaction using numerical diagonalization.

\section{B. Computing indistinguishability in the fractional quantum Hall regime}

Exact diagonalization can be used to compute reduced density matrices and therefore the indistinguishability in the FQH regime. In the occupation representation with $N_{\phi}$ orbits, the $N$-particle FQH states are given by the following general expression:

$$
|\Psi\rangle=\sum_{i}^{N_{s}} \lambda_{i}\left|N_{i}\right\rangle
$$

where $\left|N_{i}\right\rangle=c_{i_{1}}^{\dagger} \cdots c_{i_{N}}^{\dagger}|0\rangle$ is the $N$-particle basis state with orbits $i_{1}, i_{2}, \cdots, i_{N}$ occupied and $\lambda_{i}$ is the normalized amplitude of the basis state. The operator $c_{i}^{\dagger}\left(c_{i}\right)$ creates (annihilates) a fermion at the $i$ th orbit. $N_{s}$ is the size of the $N$-particle Hilbert space.

To compute the indistinguishability we must compute the $n$-particle reduced density matrix, $\rho^{(n)}$. The total $N$-particle density matrix is given by $\rho_{T} \equiv|\Psi\rangle\langle\Psi| . \rho^{(n)}$ can be computed using $\operatorname{Tr}_{N-n}\left(\rho_{T}\right)=\sum_{\alpha}\left\langle\alpha\left|\rho_{T}\right| \alpha\right\rangle$, where $\alpha$ denotes all $m$ particle basis states: $|\alpha\rangle=c_{i_{1}}^{\dagger} \cdots c_{i_{m}}^{\dagger}|0\rangle$ with $m=N-n$. The reduced density matrix can now be decomposed in the $n$-particle basis of $\left|n_{i}\right\rangle$ in the occupation representation. The reduced density matrix elements are then given by

$$
\begin{aligned}
\rho_{a, b}^{(n)} & =\left\langle n_{a}\left|\operatorname{Tr}_{m}\left(\rho_{T}\right)\right| n_{b}\right\rangle / N_{c} \\
& =\left\langle n_{a}\left|\sum_{k}^{m_{s}}\left(c_{k_{m}} \cdots c_{k_{1}}\right) \rho_{T}\left(c_{k_{1}}^{\dagger} \cdots c_{k_{m}}^{\dagger}\right)\right| n_{b}\right\rangle / N_{c} \\
& =\sum_{i, j}^{N_{s}} \sum_{k}^{m_{s}} \lambda_{i} \lambda_{j}^{*} T(a, k, i) T^{*}(b, k, j) / N_{c},
\end{aligned}
$$

where $T(a, k, i)=\left\langle n_{a}\left|c_{k_{m}} \cdots c_{k_{1}}\right| N_{i}\right\rangle, N_{c}=\left(\begin{array}{c}m \\ N\end{array}\right)$ is a normalization constant, and $m_{s}$ is the size of the Hilbert space for $m$-particle states.

For a pure system on the torus geometry, $N$-particle states $|\Psi\rangle$ can be calculated exactly in the momentum subspace. The momentum operator is given by $J_{N}=\operatorname{Mod}\left(\sum_{k=1}^{N} i_{k}, N_{\phi}\right)$ for all basis states $\left|N_{i}\right\rangle$. To get nonzero matrix elements $\rho_{a, b}^{(n)}$, we require that the $n$-particle states $\left|n_{a}\right\rangle$ and $\left|n_{b}\right\rangle$ have the same momentum $J_{n}=\operatorname{Mod}\left(J_{N}-J_{m}, N_{\phi}\right)$ with $J_{m}=$ $\operatorname{Mod}\left(\sum_{p=1}^{m} k_{p}, N_{\phi}\right)$. Noting this conservation of momentum rule, we calculate the trace norm term of Eq. (1) in momentum sub-blocks. This use of translational symmetry considerably reduces the Hilbert space size.

We compare different states using exact diagonalization on the torus and the above expressions for the indistinguishability. In the following subsections, we show results with hexagonal unit cells. We have checked that different choices for unit cells do not impact our conclusions. 


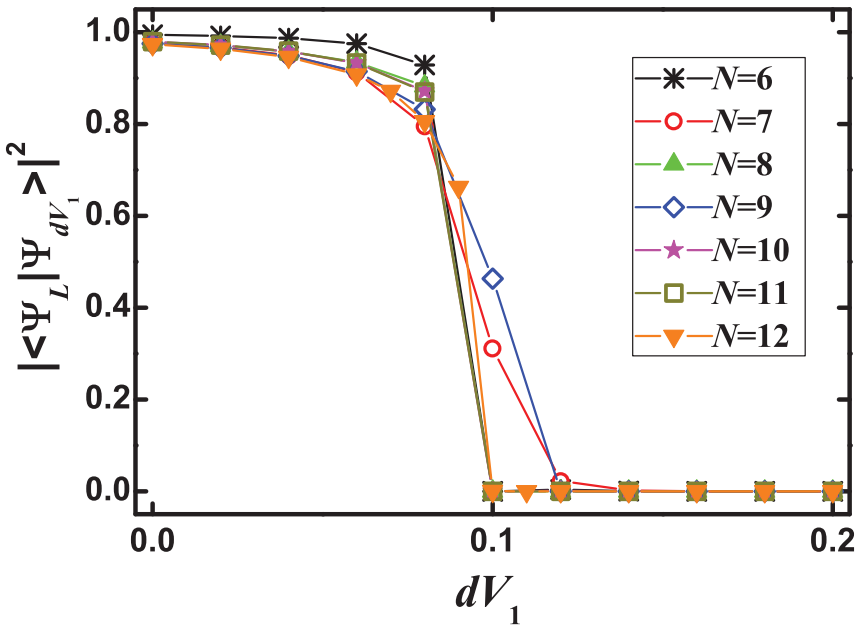

FIG. 3. (Color online) Squared wave function overlap between Laughlin state and the ground state of $v=1 / 3$ system with modulated pseudopotentials $V_{1}$.

\section{Distinguishing the Laughlin and charge density wave states}

We first compare the uniform Laughlin state with a gapless state at one-third filling. A transition between the Laughlin state and the gapless state can be driven by softening the short range part of the Coulomb interaction, $V_{1} \rightarrow V_{1}-d V_{1}$. This gapless state is a nontopological CDW. Such CDW states have been discussed in the literature ${ }^{16,28-30}$ at a variety of fillings.

Figure 3 shows the overlap between the Laughlin and the lowest energy state, $\Psi_{d V_{1}}$, from the softened Coulomb model as a function of $d V_{1}$ for several system sizes. Here we see that in the lowest LL the Coulomb point $\left(d V_{1}=0\right)$ lies squarely in the Laughlin liquid regime. But as the short ranged part of the Coulomb interaction is softened, the Laughlin gap collapses (not shown) to reveal a transition toward the CDW phase. The CDW phase is nonuniform and may occur at a momentum different from the Laughlin state. We note that there are many nearly degenerate states in the CDW regime. There is a small energy splitting in finite sized systems. We take the lowest energy state.

We now explore the nature of the liquid-to-CDW transition using the indistinguishability. The Laughlin state is a topological state with degenerate topological sectors but the CDW state does not represent a topologically ordered state. It is best described by local correlators. We therefore expect a distinct signature in $I_{n}$ in the transition.

Figure 4 shows $I_{n}$ versus $c$ for several different pseudopotentials as we cross the transition from the Laughlin liquid (top points) to the CDW state (bottom points). The data for each $d V_{1}$ represent composites from several different values of $N$ indicating that even for small $N$ we have approximate data collapse for $I_{n}$ (away from the transition point). Near the transition point $\left(d V_{1} \sim 0.10\right)$ the data scatter. From the figure we see that above the transition $\left(0 \leqslant d V_{1} \lesssim 0.08\right)$ the Laughlin state is barely distinguishable from the ground state of the softened Coulomb interaction. Once we cross the transition $\left(d V_{1} \gtrsim 0.12\right)$ the Laughlin state can be distinguished from the CDW state but only with measurements on $n \sim N$ particles. This is surprising because the CDW state is locally nonuniform

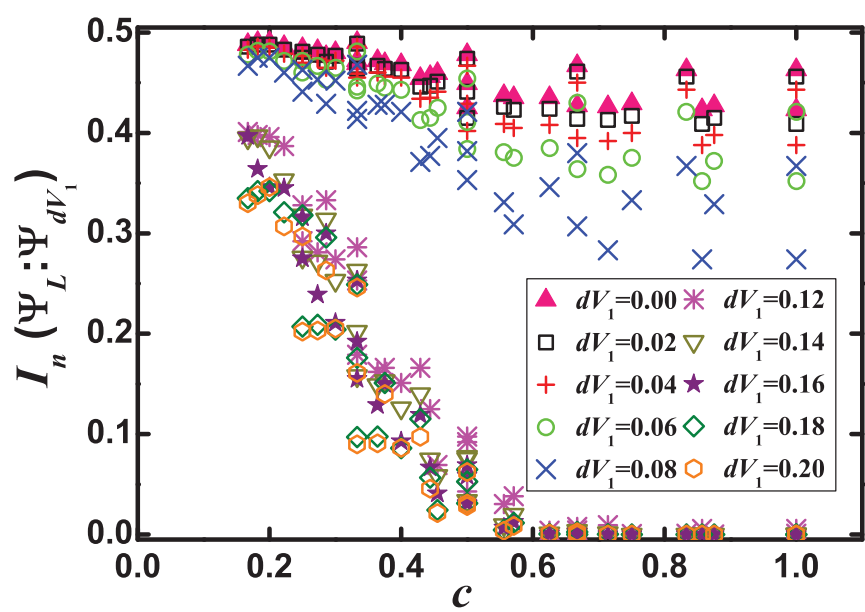

FIG. 4. (Color online) Indistinguishability between Laughlin state and calculated ground state as a function of $c$ for $v=1 / 3$ system with different pseudopotentials. The data for each $d V_{1}$ are from different system sizes $N=6,7,8,9,10,11$, and 12 .

and one would expect it to be locally different from the uniform Laughlin state. We note however that here we have compared only the lowest energy CDW state that arises in our finite size calculation. Inclusion of all low energy CDW states that arise in the thermodynamic limit may lower the $n=O(N)$ dependence to an $n=O(1)$ dependence.

\section{Distinguishing topological sectors in the fractional quantum Hall regime}

We now examine the indistinguishability between two distinct topological sectors. We first compare two Laughlin states. On the torus there are three degenerate Laughlin states. These orthogonal Laughlin states define distinct topological sectors. We now ask if two distinct sectors can be distinguished with $n=O(\sqrt{N})$ correlation functions.

In Figure 5(a) we plot the indistinguishability computed for two degenerate Laughlin states, $\Psi_{L 1}$ and $\Psi_{L 2}$. The approximate data collapse has been shown for different system sizes,

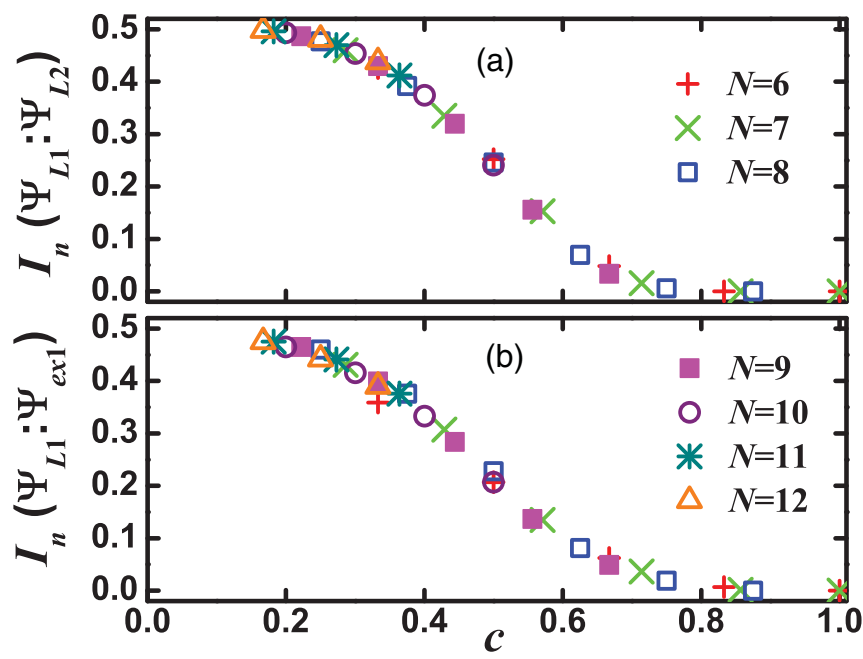

FIG. 5. (Color online) Indistinguishability as a function of $c$ for $v=1 / 3$ system (a) between two degenerate Laughlin states and (b) between Laughlin state and the first excited state. 


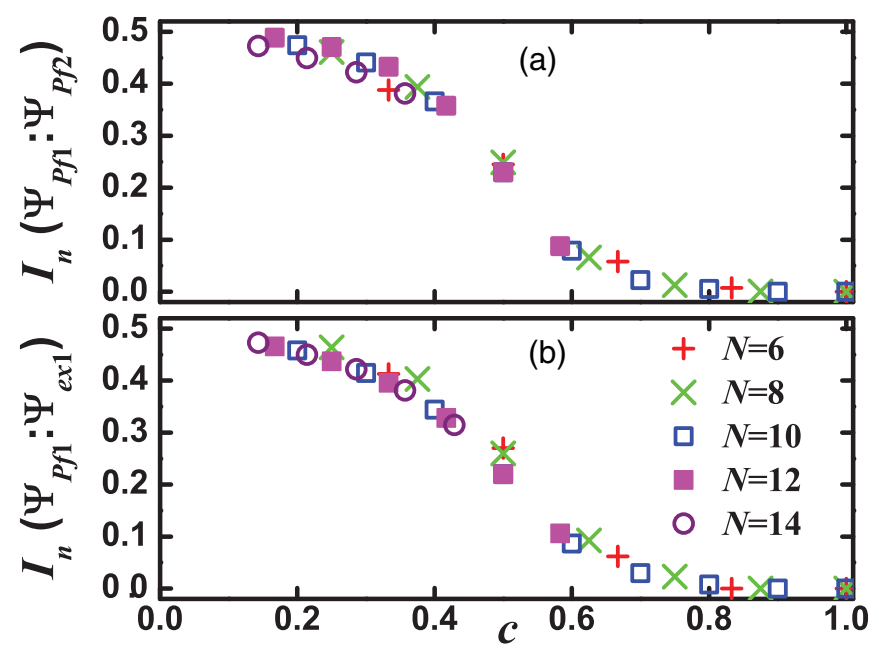

FIG. 6. (Color online) Indistinguishability as a function of $c$ for the $v=5 / 2$ system (a) between two degenerate Moore-Read states and (b) between the Moore-Read ground state and the first excited state.

indicating that the results are valid for the thermodynamic limit. We find that the two states are nearly indistinguishable for small $c$ but become distinguishable only for large $c \gtrsim 0.5$. We do not find an $n=O(\sqrt{N})$ dependence. Instead, the nonlocal, $O(N)$, distinction between states is found to be a generic feature of any two quantum Hall states. Figure 5(b) compares the Laughlin state $\Psi_{L 1}$ with its first excited state $\Psi_{\text {ex } 1}$ at zero momentum. The first excited state can be thought of as a composite fermion particle-hole pair that is formed from superpositions of all electron coordinates. ${ }^{8,31}$ We find precisely the same $I_{n}$ dependence here indicating that the structure we observe is generic for any two orthogonal lowest LL states derived from short range models. For long range interactions we find that the $I_{n}$ versus $n$ dependence exhibits the similar behavior.

We have also checked the non-Abelian Moore-Read states. From Eq. (9) we can generate six degenerate Moore-Read states on the torus, corresponding to six distinct topological sectors. We plot the indistinguishability between two distinct Moor-Read states, $\Psi_{P f 1}$ and $\Psi_{P f 2}$, in Fig. 6(a) and the indistinguishability between $\Psi_{P f 1}$ and its first excited state, $\Psi_{\mathrm{ex} 1}$, in Fig. 6(b) for different system sizes. These figures show precisely the same generic structure as Abelian Laughlin states.

\section{SUMMARY}

We have computed the indistinguishability $I_{n}$ [Eq. (1)] between distinct topological states in two different types of two-dimensional models, the toric code lattice model of spins and $\mathrm{FQH}$ models of 2DEGs in a strong magnetic field. Both models show ground states with topological degeneracies. Using $I_{n}$ we were able to show that the nature of the topological order in the toric code is distinct from that in the $\mathrm{FQH}$ regime.

In the toric code, basis states are localized in two dimensions to lie at discrete sites. The model was constructed to obey strictly one-dimensional symmetries. These symmetries then yield topological degeneracies when the model is placed on a surface with periodic boundaries. As a result our calculation of $I_{n}$ showed that measurements on a carefully chosen set of $n \sim \sqrt{N}$ spins in an $N$-particle system can accurately distinguish topological sectors, as expected. The topological quantum Hall states, in contrast, always show an $n \sim N$ dependence. This is a result of the one-dimensional nature of the Hilbert space itself. FQH correlators distinguishing two states ${ }^{10}$ span all particles even though the correlators are constructed from a one-dimensional product of operators.

Our study used the indistinguishability to show that quantum Hall states in periodic systems can only be distinguished with correlators of the order of the system size, $N$. This implies that the wave function is sufficient and necessary in a full description of FQH states. Our results also imply that in comparing candidate quantum Hall states in systems without edges, overlap is an efficient tool to distinguish two states (overlap is equivalent to $I_{n=N}$ up to a constant factor). Our results show that small-size correlation functions $(n \lesssim N / 2)$ can not accurately distinguish two quantum Hall states in the torus geometry.

It would be interesting to extend our analysis to systems with edges. Correlators of $\mathrm{FQH}$ edge states can be used to distinguish states in the bulk. ${ }^{32}$ A calculation of $I_{n}$ for systems with edges may show a different $n$ dependence for states chosen near the system edge.

\section{ACKNOWLEDGEMENT}

V.W.S. acknowledges support from the Jeffress Memorial Trust, Grant No. J-992.

\footnotetext{
${ }^{1}$ X.-G. Wen, Int. J. Mod. Phys. B 4, 239 (1990).

${ }^{2}$ A. Kitaev, Ann. Phys. (NY) 303, 2 (2003).

${ }^{3}$ X.-G. Wen and Q. Niu, Phys. Rev. B 41, 9377 (1990).

${ }^{4}$ D. I. Tsomokos, A. Hamma, W. Zhang, S. Haas, and R. Fazio, Phys. Rev. A 80, 060302(R) (2009).

${ }^{5}$ M. Haque, O. Zozulya, and K. Schoutens, Phys. Rev. Lett. 98, 060401 (2007); A. Sterdyniak, N. Regnault, and B. A. Bernevig, e-print arXiv:1006.5435 (to be published).

${ }^{6}$ R. B. Laughlin, Phys. Rev. Lett. 50, 1395 (1983).

${ }^{7}$ Perspectives in Quantum Hall Effects, edited by S. Das Sarma and A. Pinczuk (Wiley, New York, 1997).

${ }^{8}$ J. K. Jain, Composite Fermions (Cambridge University Press, Cambridge, 2007).
}

\footnotetext{
${ }^{9}$ A. Kitaev, Ann. Phys. (NY) 321, 2 (2006).

${ }^{10}$ F. D. M. Haldane, Phys. Rev. Lett. 51, 605 (1983).

${ }^{11}$ Z. Nussinov and G. Ortiz, Ann. Phys. (NY) 324, 977 (2009).

${ }^{12} \mathrm{C}$. Helstrom, Quantum Detection and Estimation Theory (Academic Press, New York, 1976).

${ }^{13}$ C. Fuchs and J. van de Graaf, IEEE Trans. Inf. Theory 45, 1216 (1999).

${ }^{14}$ J. I. Korsbakken, K. B. Whaley, J. Dubois, and J. I. Cirac, Phys. Rev. A 75, 042106 (2007).

${ }^{15}$ B. Bauer, M. Troyer, V. W. Scarola, and K. B. Whaley, Phys. Rev. B 81, 085118 (2010).

${ }^{16}$ A. A. Koulakov, M. M. Fogler, and B. I. Shklovskii, Phys. Rev. Lett. 76, 499 (1996).
} 
${ }^{17}$ G. Moore and N. Read, Nucl. Phys. B 360, 362 (1991).

${ }^{18}$ C. Castelnovo, S. Trebst, and M. Troyer, in Understanding Quantum Phase Transitions, edited by L. D. Carr (CRC Press, Boca Raton, 2010).

${ }^{19}$ S. Trebst, P. Werner, M. Troyer, K. Shtengel, and C. Nayak, Phys. Rev. Lett. 98, 070602 (2007).

${ }^{20}$ L. Tagliacozzo and G. Vidal, e-print arXiv:1007.4145 (to be published).

${ }^{21}$ M. S. Watanabe, Phys. Rev. E 51, 3945 (1995).

${ }^{22}$ D. Stauffer and A. Aharony, Introduction to Percolation Theory (Taylor \& Francis, London, 1994).

${ }^{23}$ F. D. M. Haldane, Phys. Rev. Lett. 55, 2095 (1985).
${ }^{24}$ M. Greiter, X.-G. Wen, and F. Wilczek, Phys. Rev. Lett. 66, 3205 (1991).

${ }^{25}$ E. H. Rezayi and F. D. M. Haldane, Phys. Rev. Lett. 84, 4685 (2000). ${ }^{26}$ M. Stone, Quantum Hall Effect (World Scientific, London, 1992).

${ }^{27}$ J. Zak, Phys. Rev. Lett. 79, 533 (1997).

${ }^{28}$ F. D. M. Haldane and E. H. Rezayi, Phys. Rev. Lett. 54, 237 (1985).

${ }^{29}$ S. Y. Lee, V. W. Scarola, and J. K. Jain, Phys. Rev. Lett. 87, 256803 (2001); Phys. Rev. B 66, 085336 (2002).

${ }^{30}$ N. Shibata and D. Yoshioka, J. Phys. Soc. Jpn. 73, 2169 (2004).

${ }^{31}$ J. K. Jain, Phys. Rev. Lett. 63, 199 (1989).

${ }^{32}$ X.-G. Wen, Phys. Rev. B 41, 12838 (1990). 